\title{
INCREASED EXTRACELLULAR MATRIX METALLOPROTEINASE INDUCER (EMMPRIN) EXPRESSION IN PULMONARY FIBROSIS
}

Stéphanie Guillot $\square$ INSERM U620, Université de Rennes 1, Rennes cedex, France; and Service de Physiologie, CHU de Rennes, Hôpital Pontchaillou, Rennes cedex, France

Philippe Delaval $\square$ INSERM U620, Université de Rennes 1, Rennes cedex, France; and Service de Pneumologie, CHU de Rennes, Hôpital Pontchaillou, Rennes cedex, France

Graziella Brinchault $\quad \square$ Service de Pneumologie, CHU de Rennes, Hôpital Pontchaillou, Rennes cedex, France

Sylvie Caulet-Maugendre $\square$ INSERM U620, Université de Rennes 1, Rennes cedex, France; and Service d'Anatomie et Cytologie Pathologiques, CHU de Rennes, Hôpital Pontchaillou, Rennes cedex, France

Alexandra Depince $\square$ INSERM U620, Université de Rennes 1, Rennes cedex, France Hervé Lena $\square$ Service de Pneumologie, CHU de Rennes, Hôpital Pontchaillou, Rennes cedex, France

Bertrand Delatour $\square$ Service de Chirurgie Thoracique et Cardiovasculaire, CHU de Rennes, Hôpital Pontchaillou, Rennes cedex, France

Vincent Lagente $\quad \square$ INSERM U620, Université de Rennes 1, Rennes cedex, France

Corinne Martin-Chouly $\square \quad$ INSERM U620, Université de Rennes 1,

Rennes cedex, France

Extracellular matrix metalloproteinase inducer (EMMPRIN) was examined on bronchoalveolar lavage fluids (BALFs) and lung tissue from patients with fibrosis (usual interstitial pneumonia-idiopathic pulmonary fibrosis [UIP-IPF], $n=15$; diffuse parenchymal lung diseases without IPF characteristics on computerized tomography scan, $n=8$ ) and without fibrosis $(n=6)$. In UIP-IPF, EMMPRIN staining was increased in areas of fibrosis, mainly in

This work is supported by a research grant from Conseil Regional de Bretagne, France (ref. no. 2004797) and a collaboration project INSERM/FIOCRUZ. The authors are thankful to Dr. Paul Baldwin for his assistance.

Address correspondence to Pr. Vincent Lagente, INSERM U620, Université de Rennes 1, 2 avenue du Pr. Léon Bernard, 35043 Rennes cedex, France. E-mail: vincent.lagente@univ-rennes1.fr 
macrophages and in epithelial cells. EMMPRIN was also found in the extracellular medium with significant levels in patients with lung fibrosis compared to subjects without fibrosis. Moreover, macrophages from patients with lung fibrosis spontaneously produce EMMPRIN. These findings show that EMMPRIN is increased in lung fibrosis.

Keywords bronchoalveolar lavage, EMMPRIN, idiopathic pulmonary fibrosis, pulmonary fibrosis, usual interstitial pneumonia

Idiopathic pulmonary fibrosis (IPF) and more generally interstitial and fibrotic lung diseases are characterized by varying degrees of inflammation and fibrosis in the lung parenchyma [1-5]. It has been previously showed that the molecular mechanisms behind the tissue remodeling and the abnormal wound healing in IPF are known to involve various proteases including matrix metalloproteinases (MMPs), a family of zinc-dependent endoproteinases [6-8]. Moreover, several studies involve the MMPs in the development of tissue remodeling and in fibrosis associated with various inflammatory conditions [8-10].

Extracellular matrix metalloproteinase inducer (EMMPRIN), also called basigin in mouse, is a transmembrane glycoprotein that belongs to immunoglobulin superfamily [11-13]. It was originally isolated from the LX-1 human pulmonary carcinoma cell line and termed tumor cell-derived collagenase stimulatory factor (TCSF) because it stimulated matrix metalloproteinases production in adjacent neighbouring normal fibroblasts, thereby enhancing the migration of malignant cells through the extracellular matrix, suggesting an important role in tumor progression [13, 14]. Besides the presence of EMMPRIN in lung carcinomas [15, 16], its up-regulation has also been shown in other malignant diseases like breast carcinomas [15-17], laryngeal carcinomas [18], and in malignant pigment cell lesions $[19,20]$. Moreover, the presence of EMMPRIN in nontumoral tissues suggests a role in other physiological and pathological situations, which may be associated with increased MMP expression. In this context, EMMPRIN is expressed in a variety of embryonic and normal adult tissues $[12,16,20]$ and was found in nonmalignant processes such as human atheroma [21], left ventricular myocardium failure [22], in ventilatorinduced lung injury in rats [23], and in normal and ulcerated corneas [24]. A recent study reported the increased EMMPRIN in bronchoalveolar lavage fluid (BALF) of smokers [25]. Furthermore, it was shown that EMMPRIN was expressed in experimental bleomycin-induced fibrosis in mice [26]. EMMPRIN has been shown to stimulate mesenchymal cell production of MMP-1 (collagenase), MMP-2 (gelatinase A), MMP-3 (stromelysin-1), MMP-9 (gelatinase B), and MMP-14 [12, 17, 27-31]. However the mechanisms by which EMMPRIN induces MMP production have not been fully established [13, 17, 28-31]. 
Because some MMPs are induced during wound healing processes in IPF and in various interstitial and fibrotic lung diseases associated with inflammatory conditions such as sarcoidosis, we hypothesized that EMMPRIN may be involved in tissue remodeling in these disorders. The aim of the present study was to evaluate the expression of EMMPRIN in BALF and in lung tissue from patients with or without interstitial and fibrotic lesions and to precise the cellular sources of EMMPRIN and the relationships between EMMPRIN and fibrosis. Thereafter, we were interested in the production of EMMPRIN by alveolar macrophages (AMs) recovered from BAL of patients with or without interstitial and fibrotic lung disorders.

\section{MATERIALS AND METHODS}

\section{Characteristics of Patients}

The study population consisted of 3 groups and their characteristics are summarized in Table 1A. The characteristics of the fourth group containing patients who were not included in the 3 initial groups but on whom immunohistochemistry experiments was performed on lung tissue are presented in Table 1B.

Group 1 is a group of usual insterstitial pneumonia (UIP)-IPF patients in accordance with the American Thoracic Society/European Respiratory Society (ATS/ERS) published norms [2, 3]. Fifteen patients with IPF (11 men and 4 women; mean age $71 \pm 2$ years; 2 smokers and 13 nonsmokers; only one had $20 \mathrm{mg}$ per day of prednisone) were included. All of them had progressive dyspnea, bilateral dry crackles, and functional findings showing a reduced total lung capacity, a decreased single-breath carbon monoxide diffusion capacity, and a resting hypoxemia or an exercise-induced hypoxemia. The UIP-IPF characteristics on computerized tomography scan were:

-presence of reticular abnormality and or traction bronchiectasies with basal and peripheral predominance;

-presence of honeycombing with basal and peripheral predominance;

-absence of atypical features as micronodules, peribronchovascular nodules, mediastinal adenopathy;

- ground glass attenuation, if present, is less extensive than reticular opacity.

Histologic confirmation of the diagnosis was obtained in 6 patients by video-assisted thoracoscopic lung biopsy showing a histologic pattern of UIP.

Group 2 is a group of patients with diffuse parenchymal lung disorders (DPLD) associated with various diseases. These cases should be distinguishable from UIP-IPF with any relevant cause. Their computerized 
TABLE 1A Characteristics of the Patients

\begin{tabular}{|c|c|c|c|c|c|}
\hline Patient & Diagnosis & $\begin{array}{l}\text { Age } \\
\text { (years) }\end{array}$ & Sex & $\begin{array}{c}\text { Tobacco } \\
\text { use }\end{array}$ & $\begin{array}{c}\text { Immunosuppressive } \\
\text { treatment }\end{array}$ \\
\hline 1 & IPF & 69 & $\mathrm{M}$ & No & No \\
\hline 2 & IPF & 75 & $\mathrm{~F}$ & No & No \\
\hline 3 & IPF & 69 & $\mathrm{M}$ & No & No \\
\hline 4 & IPF & 70 & $\mathrm{M}$ & No & No \\
\hline 5 & IPF & 59 & M & No & No \\
\hline 6 & IPF & 74 & $\mathrm{~F}$ & No & No \\
\hline 7 & IPF & 65 & M & No & No \\
\hline 8 & IPF & 74 & $\mathrm{M}$ & Yes & No \\
\hline 9 & IPF & 74 & M & No & No \\
\hline 10 & IPF & 59 & M & No & $\begin{array}{l}20 \mathrm{mg} \text { per day of prednisone } \\
\text { for } 7 \text { months }\end{array}$ \\
\hline 11 & IPF & 79 & M & No & No \\
\hline 12 & IPF & 70 & $\mathrm{~F}$ & No & No \\
\hline 13 & IPF & 79 & $\mathrm{~F}$ & No & No \\
\hline 14 & IPF & 77 & $\mathrm{M}$ & No & No \\
\hline 15 & IPF & 58 & M & Yes & No \\
\hline 16 & DPLD scleroderma & 48 & $\mathrm{~F}$ & No & No \\
\hline 17 & DPLD lupus & 36 & $\mathrm{~F}$ & No & $\begin{array}{l}9 \mathrm{mg} \text { per day of prednisone } \\
\text { since } 1989\end{array}$ \\
\hline 18 & $\begin{array}{l}\text { DPLD bronchiolitis obliterans } \\
\text { organizing pneumonia }\end{array}$ & 43 & M & No & No \\
\hline 19 & DPLD scleroderma & 68 & F & Yes & No \\
\hline 20 & DPLD rhumatoid arthritis & 64 & M & No & $\begin{array}{l}10 \mathrm{mg} \text { per day of prednisone } \\
\text { since } 1996\end{array}$ \\
\hline 21 & DPLD sarcoidosis & 66 & $\mathrm{~F}$ & No & No \\
\hline 22 & $\begin{array}{l}\text { DPLD hypersensitivity } \\
\text { pneumonitis }\end{array}$ & 48 & M & No & No \\
\hline 23 & DPLD sarcoidosis & 50 & M & No & No \\
\hline 24 & Non-DPLD lung cancer & 55 & M & Yes & No \\
\hline 25 & Non-DPLD gut cancer & 53 & M & No & No \\
\hline 26 & Non-DPLD pulmonary nodule & 64 & $\mathrm{~F}$ & No & No \\
\hline 27 & Non-DPLD alveolar collapse & 55 & M & Yes & No \\
\hline 28 & Non-DPLD cardiopathy & 53 & $\mathrm{~F}$ & No & $\begin{array}{l}20 \mathrm{mg} \text { per day of prednisone } \\
\text { for } 8 \text { months }\end{array}$ \\
\hline 29 & Non-DPLD tracheal stenosis & 35 & $\mathrm{~F}$ & Yes & No \\
\hline
\end{tabular}

Note. IPF = idiopathic pulmonary fibrosis; DPLD = diffuse parenchymal lung disorder; $\mathrm{M}=$ male; $\mathrm{F}=$ female.

tomography scans have a common characteristic of interstitial and/or fibrotic pattern without IPF characteristics. Any patient of this group had lung biopsy to exactly determine the histologic pattern of fibrosis. Even if the most prevailing pattern in some collagen vascular diseases and scleroderma has been shown to be UIP, this one should be distinguishable from the IPF/UIP pattern, as found in the patients of the first group. Eight patients ( 4 men and 4 women; mean age $53 \pm 4$ years; 1 smoker and 7 nonsmokers; two of them were under steroids for many years) were included in this group. All patients had pulmonary infiltrates and five of them had 
TABLE 1B Characteristics of the Patients on Whom Immunohistochemistry Studies on Lung Parenchyma Were Performed

\begin{tabular}{llcccc}
\hline Patient & \multicolumn{1}{c}{ Diagnosis } & Age (years) & Sex & Tobacco use & $\begin{array}{c}\text { Immunosuppressive } \\
\text { treatment }\end{array}$ \\
\hline 30 & Sarcoidosis & 40 & $\mathrm{M}$ & No & No \\
31 & Sarcoidosis & 36 & $\mathrm{M}$ & No & No \\
32 & Sarcoidosis & 34 & $\mathrm{M}$ & No & No \\
33 & Sarcoidosis & 64 & $\mathrm{~F}$ & No & No \\
34 & Sarcoidosis & 56 & $\mathrm{M}$ & No & No \\
35 & Dystrophic bullous disease & 37 & $\mathrm{~F}$ & Yes & No \\
36 & Dystrophic bullous disease & 22 & $\mathrm{M}$ & Yes & No \\
37 & Dystrophic bullous disease & 18 & $\mathrm{M}$ & No & No \\
38 & Dystrophic bullous disease & 27 & $\mathrm{M}$ & Yes & No \\
39 & Dystrophic bullous disease & 16 & $\mathrm{M}$ & No & No \\
40 & Lung tumor & 63 & $\mathrm{~F}$ & No & No \\
41 & Lung tumor & 51 & $\mathrm{M}$ & Yes & No \\
42 & Lung tumor & 77 & $\mathrm{M}$ & Yes & No \\
43 & Lung tumor & 47 & $\mathrm{M}$ & Yes & \\
\hline
\end{tabular}

Note. $\mathrm{M}=$ male; $\mathrm{F}=$ female.

evidence of fibrosis (honeycombing and/or traction bronchiectasies) on computerized tomography scan.

The group 3 consists of six patients without diffuse parenchymal lung disorders (non DPLD) but not healthy subjects, because of the difficulty of having BAL in healthy subjects (3 men and 3 women; mean age $53 \pm 4$ years; 3 smokers and 3 nonsmokers; one had $20 \mathrm{mg}$ per day of prednisone). These patients had no radiological pulmonary infiltrates.

Group 4 consists of patients who had lung biopsy or lung surgery and permited us to do immunohistochemistry on their lung parenchyma. Indeed, lung parenchyma was not available for patients from groups 2 and 3 (no medical indication of doing pulmonary biopsy in the third group) compared with some patients from the first group who had lung biopsy $(n=6)$. Among these patients, we included some with diffuse parenchymal lung disorders due to sarcoidosis $(n=5,4$ men and 1 woman; mean age $46 \pm 6$ years; all nonsmokers; without immunosuppressive drugs). Moreover, we obtained normal lung tissue from patients who had lung surgery for subpleural dystrophic fibroemphysematous lesions ( $\mathrm{n}=5$, 4 men and 1 woman; mean age $24 \pm 4$ years; 3 smokers and 2 nonsmokers; without immunosuppressive drugs) or for primary lung tumor ( $\mathrm{n}=4$, 3 men and 1 woman; mean age $60 \pm 7$ years; 3 smokers and 1 nonsmoker; without immunosuppressive drugs). In these 9 cases, lung tissue was taken at a distant site from the pathological process, and had normal structure, therefore these slides were used as control specimens.

This study was approved by the ethical committee of human subjects of the hospital of Rennes (France). Informed consent was obtained from all patients. 


\section{Bronchoalveolar Lavages (BALs)}

BALs were performed under local anesthesia with 2\% lidocaine. BALs were obtained from 12 IPF patients and for all other patients. A fiberoptic bronchoscope was wedged into the segmental bronchus of the middle lobe or into the lingula. Sterile $0.9 \%$ saline totaling 100 to $150 \mathrm{~mL}$ was instilled in aliquots of $50 \mathrm{~mL}$ and recovered into sterile propylene tubes. For the patient with lung cancer (patient no. 24, Table 1A), the BAL was made in the contralateral lung at distance from the carcinoma. The different aliquots were pooled. Mucus was filtered through sterile gauze. Then, lavage fluid was centrifuged at $481 \times g$ for 7 minutes at $6^{\circ} \mathrm{C}$ to separate cells from cellfree-fluid. The cell-free lavage was stored at $-80^{\circ} \mathrm{C}$ until further analysis.

\section{Pulmonary Biopsies}

The lung sections were fixed in $10 \%$ formalin, embedded in paraffin, and sectioned with a cryostat at a thickness of $5 \mu \mathrm{m}$. For histological studies, sections were stained with hematoxylin and eosin.

\section{Immunohistochemistry}

Immunohistochemical studies were performed using polyclonal primary antibody directed against the N-terminal region of the human EMMPRIN (rabbit anti-EMMPRIN; Zymed Laboratories, San Francisco, USA). To localize EMMPRIN, immunohistochemistry was prepared on paraffin-embedded tissue with a standard UltraTech horseradish peroxidase (HRP)-streptavidin-biotin system (Immunotech, Marseille, France) according to the manufacturer's instructions, using an automate stainer (Dako, France). Briefly, after deparaffinization and rehydration in toluene and alcohols, the sections were washed with phosphate-buffered saline (PBS). Endogenous peroxidase activity was eliminated by incubation with $0.3 \%$ hydrogen peroxide $\left(\mathrm{H}_{2} \mathrm{O}_{2}\right)$ at room temperature for 15 minutes. This was followed by incubation in a moist chamber with the primary antibody (polyclonal rabbit primary antibody directed against the N-terminal region of the human EMMPRIN (1:100) for 60 minutes at room temperature. This antibody is intended to qualitatively stain the EMMPRIN antigen and demonstrated cell membrane staining throughout the normal epithelium, as well as membrane staining in various carcinomas. After being washed with PBS, the sections were incubated with biotinylated secondary antibody for 15 minutes and they were then washed and incubated for 15 minutes with streptavidin-hydroxyperoxidase complex. Finally, the immunoreaction was revealed by incubation with diaminobenzidine tetrahydrochloride chromogen, counterstained with hematoxylin solution, and mounted. Slides that were not exposed to the primary antibody were used as negative 
controls. The intensity of the staining in different lung tissue components was evaluated using a scale from 0 to $+++: 0=$ negative; $+=$ mild; $++=$ moderate; and $+++=$ strong staining.

\section{Evaluation of EMMPRIN by Western Blotting}

Three microgram of protein of each sample were mixed with $10 \mu \mathrm{L}$ of a solution containing Tris $250 \mathrm{mM} \mathrm{pH} 6.8$, sucrose $4 \%$ (Fluka Biochemika, Buchs, Switzerland), sodium dodecyl sulfate (SDS) 10\% (Eurobio, Les Ulis, France), bromophenol blue $0.18 \%$ (Sigma, St Louis, USA), and $\beta_{2}$ mercaptoethanol $2 \%$ (Sigma). Then the slides were boiled for 10 minutes at $100^{\circ} \mathrm{C}$, separated on a $10 \%$ SDS-polyacrylamide gel, and transferred to a nitrocellulose filter (Hybond ECL; Pharmacia Biotech, Amersham, UK) overnight at $4^{\circ} \mathrm{C}$. The membrane was stained with ponceau red to show protein loading and transfer completeness. Then, the membrane was blocked in 3\% milk in Tris-buffered saline (TBS) for 2 hours at room temperature before exposure for the same time with the primary antibody (N-terminal specific EMMPRIN goat polyclonal antibody, 1:500; R\&D Systems, Abingdon, UK) diluted in $2 \%$ milk in a solution containing TBS, NP40 $0.3 \%$ (Sigma), and sodium chloride $0.1 \mathrm{M}$. The membranes were then incubated for 2 hours at room temperature with a secondary rabbit anti-goat-peroxidase conjugate (1:2000, R\&D Systems) diluted in 2\% milk in a solution containing TBS, NP40 $0.3 \%$, and sodium chloride $0.1 \mathrm{M}$. Immunoreactive protein bands were detected with electrochemiluminescence. Enzyme levels were quantified by measuring the intensity of the bands after scanning of the membrane using a densitometric analyzer (BIO-PROFIL-1D) and results were expressed as a percentage of the band intensity of a common reference sample loaded onto each gel.

\section{Cell Culture}

To evaluate the production of EMMPRIN by alveolar macrophages (AMs), AM cultures recovered from BAL were performed. The viability of the BAL cells, as assessed by trypan blue exclusion, was always $>85 \%$. An aliquot of BAL cells was subjected to a differential count by using Giemsa-stained cytocentrifuge preparation and AMs were considered to represent a pure population of cells if the macrophages comprised $>85 \%$ of the total cells recovered by lavage. The BAL cells were resuspended in RPMI 1640 medium with glutamax (Gibco) supplemented with $10 \%$ heat-inactivated fetal calf serum, $100 \mathrm{UI} / \mathrm{mL}$ penicillin, and $100 \mu \mathrm{g} / \mathrm{mL}$ streptomycin. The cell suspension was added at a final known concentration in plastic tissue culture plates (Falcon; Becton Dickinson, Franklin Lakes, USA). AMs were isolated by adhesion to culture plates over 
1 hour at $37^{\circ} \mathrm{C}$. The nonadherent cells were removed by 3 washes with culture medium. The purified AMs were incubated for an additional 24 hours with RPMI 1640 medium with glutamax. The culture supernatants of the AMs were harvested at successive times and were stored in fractions at $-80^{\circ} \mathrm{C}$ until analysis. The cells were solubilized with $150 \mu \mathrm{L}$ of lysis buffer ( $10 \%$ sodium dodecyl sulfate, $4 \%$ sucrose, and $250 \mathrm{mM}$ Tris- $\mathrm{HCl} \mathrm{pH} 6.8)$. Cellular lysates were then harvested and stored until analysis. Protein concentrations were measured by using the Bradford reagent (BioRad) on the slides. Western blot analysis for EMMPRIN were performed on culture supernatants and cell lysis.

\section{Statistical Analysis}

Values were expressed as means \pm SEM. Statistical analysis were performed on the data using non parametric tests. Kruskal-Wallis test was used to compare the distribution of EMMPRIN between the different groups. The Mann-Whitney test was applied to compare differences between two groups. The correlations between the expression of EMMPRIN and cell counts were analyzed using Spearman's rank correlation test. The correlations between EMMPRIN expression and tobacco use were analyzed using a Student's $t$ test. In all tests, $P$ values $<.05$ were considered statistically significant.

\section{RESULTS}

\section{EMMPRIN Expression in Lung Tissue}

Specific immunoreactivity for EMMPRIN was observed on lung fibrotic tissue obtained from patients of IPF group $(n=6)$ and from subjects from the fourth group who had lung fibrosis due to sarcoidosis $(n=5)$. As control specimens, we used normal lung parenchyma at distance from the pathological process from patients who had lung surgery for fibroemphysematous lesions or for primary lung tumor $(n=9)$. The data are presented in Table 2 and Figure 1.

In UIP pattern, EMMPRIN staining was increased in the areas of endstage fibrosis with honeycomb change and areas of active fibrosis with fibroblastic foci compared to the regions of normal lung. First, EMMPRIN was mainly localized in alveolar and tissue macrophages with a clearly underlined plasmic membrane and a weaker diffuse cytoplasmic staining, as well in the areas with and without fibrosis, but with a stronger intensity in the abnormal fibrotic regions (Figure 1A,B). EMMPRIN was also expressed by type II pneumocytes at the edges of the fibrotic areas, in contact of fibroblastic foci and in the honeycomb areas. Staining was observed underlining the cellular membrane in contact with the basement matrix of the alveolar 
TABLE 2 EMMPRIN Immunoreactivity in Lung Tissue with a Pattern of Usual Interstitial Pneumonia (UIP) $(\mathrm{n}=6)$, Sarcoidosis $(\mathrm{n}=5)$, and Normal Lung Parenchyma $(\mathrm{n}=9)$

\begin{tabular}{lcccc}
\hline & \multicolumn{2}{c}{ UIP } & & \\
\cline { 2 - 4 } Site & $\begin{array}{c}\text { Areas of } \\
\text { active fibrosis }\end{array}$ & $\begin{array}{c}\text { Areas without } \\
\text { fibrosis }\end{array}$ & Sarcoidosis & $\begin{array}{c}\text { Normal lung } \\
\text { parenchyma }\end{array}$ \\
\hline Alveolar macrophages & +++ & ++ & + & + \\
Tissue macrophages & ++ & + & +++ & 0 \\
Alveolar epithelial cells & ++ & & (in granulomas) & 0 \\
Bronchiolar epithelial cells & ++ & 0 & 0 & + \\
Interstitial fibroblasts and & 0 & + & 0 & 0 \\
$\quad$ myofibroblasts & + & 0 & 0 & 0 \\
Lymphocytes & 0 & 0 & 0 & 0 \\
Vascular smooth muscle cells & 0 & 0 & 0 & 0 \\
Airway smooth muscle cells & 0 & 0 & 0 & 0 \\
Endothelial cells & & 0 & 0 & 0 \\
\hline
\end{tabular}

Note. $\mathrm{n}=$ the number of patients; UIP $=$ usual interstitial pneumonia; $0=$ no staining; $+=$ weak staining; $++=$ moderate staining; $+++=$ strong staining.

spaces and between cells (Figure 1A). Moreover, bronchiolar cells on UIP sections displayed a diffuse expression of EMMPRIN (Figure 1C). Lung IPF sections performed without the primary antibody did not show staining (Figure 1D). Inflammatory components as small lymphocytes, when present, demonstrated weak positive staining. In preserved areas, bronchiolar cells and alveolar macrophages were positive whereas type I and II peumocytes were negative (Figure $1 B$ ). Fibroblasts, smooth muscle, and endothelial cells were negative on all UIP sections.

In sarcoidosis, we also observed a high staining of tissular activated macrophages in granulomas and a less intense reaction in alveolar macrophages (Figure 1E, F). Moreover, bronchiolar epithelial cells were also stained (Figure $1 F$ ). Pneumocytes showed no reaction either in the areas of active inflammation regarding granulomas or in areas of fibrosis (Figure $1 F$ ).

In normal lung, only macrophages and bronchiolar epithelial cells were positive with a moderate staining whereas pneumocytes did not expressed EMMPRIN (Figure $1 G$ ). Lung sections performed without the primary antibody were not stained (Figure $1 H$ ).

Moreover, there was no EMMPRIN staining within any lung slide on interstitial mesenchymal cells like fibroblasts and myofibroblasts, on vascular smooth muscle cells, airway smooth muscle cells, and endothelial cells.

\section{Western Blot Analysis of EMMPRIN Presence in BAL Fluids}

Western blot analysis for EMMPRIN demonstrated an identical electrophoretic profile with the presence of a $57 \mathrm{kDa}$ form in all BAL fluids 
A

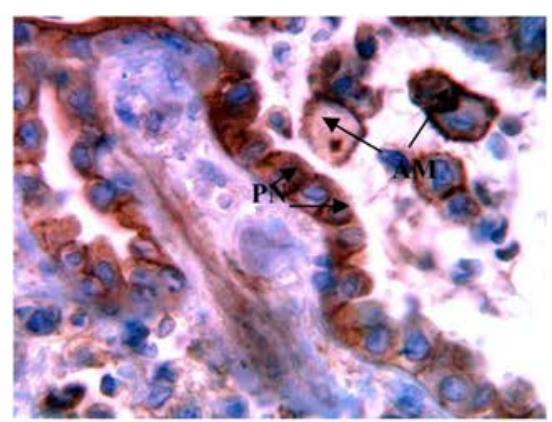

C

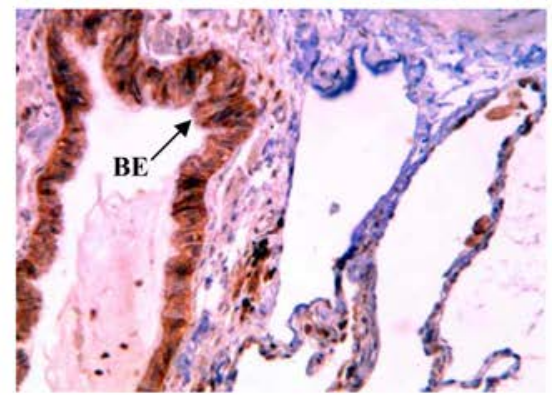

E

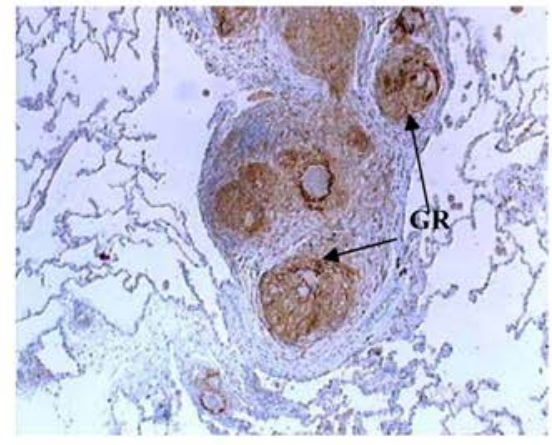

G

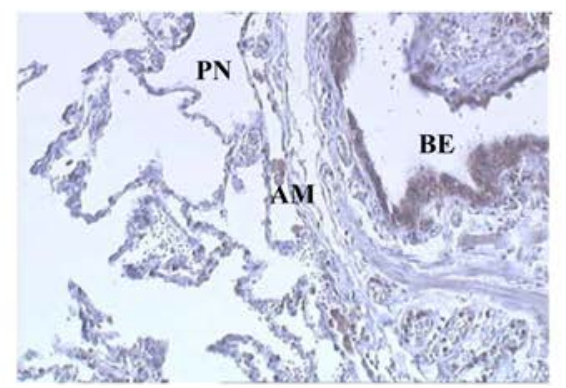

B

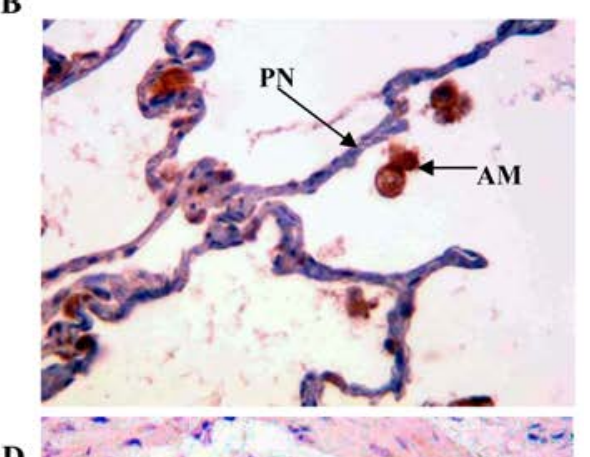

D :

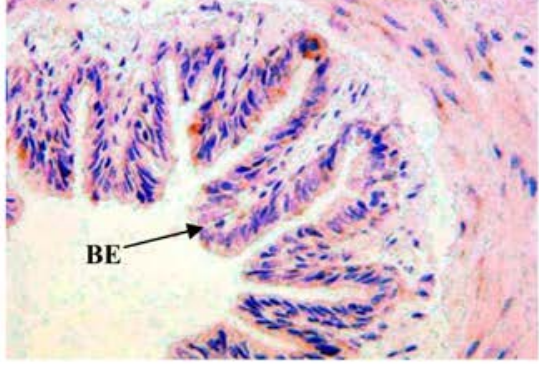

F

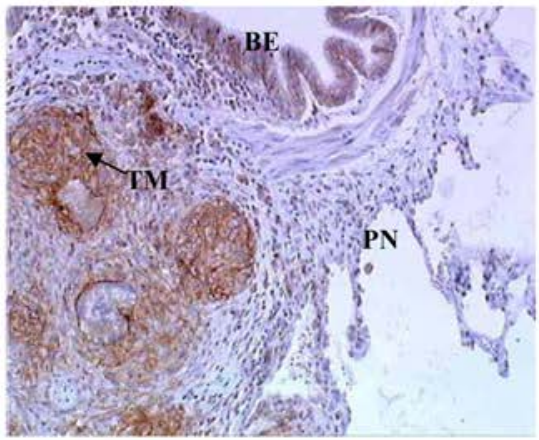

H

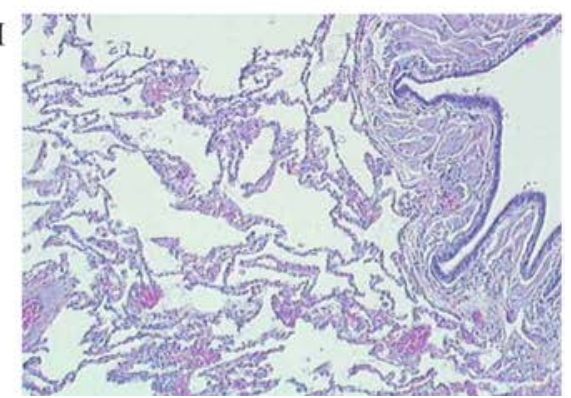


evaluated (IPF, $\mathrm{n}=12$; DPLD, $\mathrm{n}=8$; without DPLD, $\mathrm{n}=6$ ) (Figure 2A). Densitometric analysis of this form indicated that EMMPRIN levels in samples from patients with IPF and with DPLD were markedly increased and differed significantly from patients without DPLD $(P<.05$ and $P<.01$, respectively) (Figure $2 B$ ). Four of the five patients with evidence of lung fibrosis on computed tomography had high levels of EMMPRIN in their BAL fluid. However, there is no relation between EMMPRIN level in BAL fluids and the immunosuppressive treatment of the patients. Moreover, no correlation between the number of alveolar macrophages of all patients tested and EMMPRIN expression in their BAL was observed. We did not found any significant difference $(P=.07)$ between the expression of EMMPRIN in smokers (mean of expression of EMMPRIN 60.8\% $\pm 31.3 \%$ ) and in nonsmoker patients (mean of expression of EMMPRIN $82.1 \% \pm$ $22.2 \%)$.

\section{Western Blot Analysis of EMMPRIN Production by Alveolar Macrophages}

In alveolar macrophage (AM) culture media from different patients, the same band at $57 \mathrm{kDa}$ was present (Figure 3). In AMs from IPF patients, we observed the presence of EMMPRIN in their culture media, suggesting the release of EMMPRIN by these cells. In the same context, EMMPRIN was also produced by AMs from a patient with DPLD (sarcoidosis), both in the culture media and in the cells themselves, whereas it was slightly found in AMs from a patient without DPLD (tracheal stenosis). Thereby, AMs from interstitial and fibrotic lung could produce more EMMPRIN than from nonfibrotic lung.

\footnotetext{
FIGURE 1 Immunolocalization of EMMPRIN in human lung parenchyma. $A-D$ : Histologic patterns of usual interstitial pneumonia. A: Areas of active fibrosis. EMMPRIN-positive type II pneumocytes (PN) and alveolar macrophages (AM). The staining of alveolar macrophages and pneumocytes is cytoplasmic with a reinforcement along the basement membrane (magnification, $\times 400)$. B: Pneumocytes $(\mathrm{PN})$ show no reaction in the areas of preserved normal lung parenchyma whereas alveolar macrophages $(\mathrm{AM})$ are stained (magnification, $\times 250)$. C: EMMPRIN-positive bronchiolar epithelial cells $(\mathrm{BE})$ (magnification, $\times 250)$. D: Control sections without the anti-EMMPRIN primary antibody. Bronchiolar epithelial cells $(\mathrm{BE})$ are not stained (magnification, $\times 400) . E-F$. Histologic patterns of pulmonary sarcoidosis. E: An intense reaction is seen in granulomas (GR) (magnification, $\times 100)$. F: Tissue macrophages (TM) in granulomas are strongly stained whereas adjacent pneumocytes (PN) are unreactive. Bronchiolar epithelial cells $(\mathrm{BE})$ show a diffuse reaction (magnification, $\times 250)$. $G-H$ : Sections of normal lung parenchyma. $G$ : Bronchiolar epithelial cells (BE) and alveolar macrophages (AM) show a moderate diffuse reaction whereas pneumocytes $(\mathrm{PN})$ are unreactive (magnification, $\times 250$ ). $H$ : Control sections without the anti-EMMPRIN primary antibody. No reaction is seen on different cellular types (magnification, $\times 250$ ).
} 


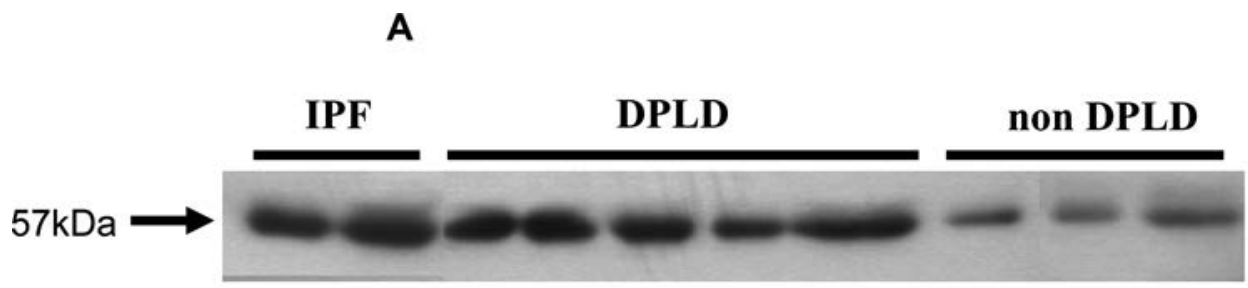

B

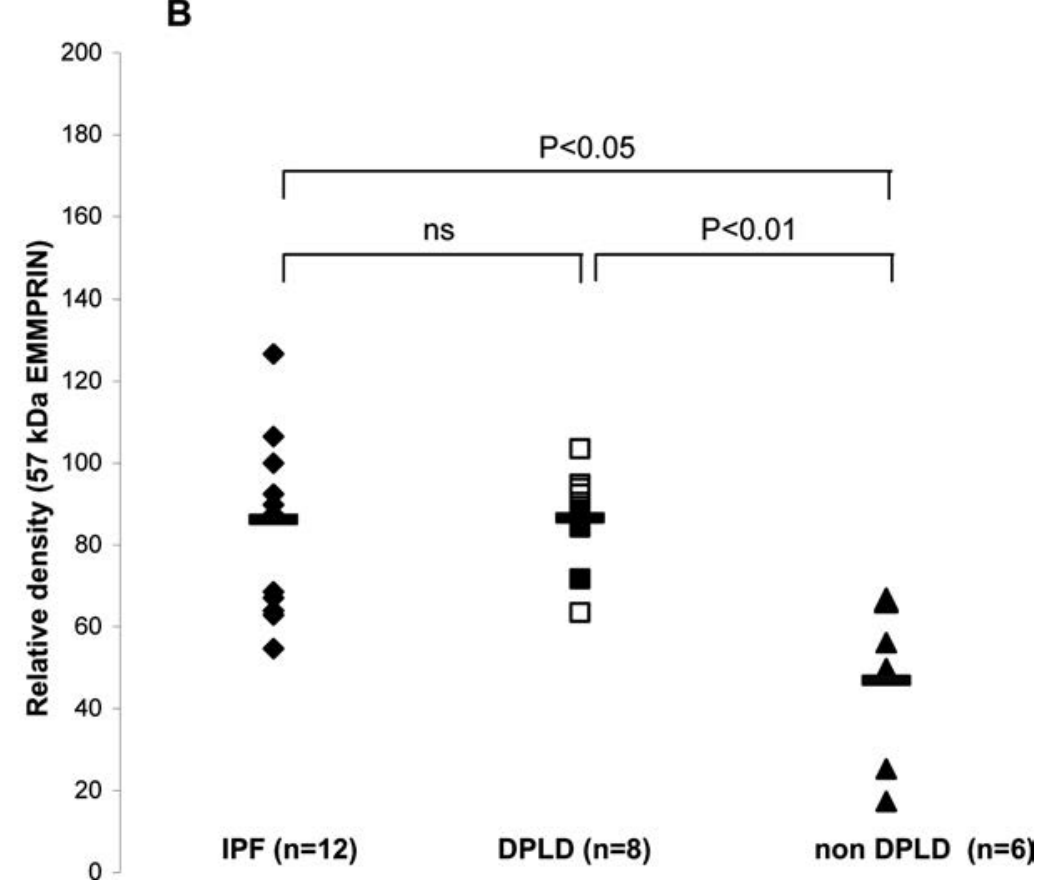

FIGURE 2 Detection of EMMPRIN by western blotting in bronchoalveolar lavages fluids of patients. (A) A blot with slides from 10 patients is presented. The anti-EMMPRIN antibody recognizes a band of $57 \mathrm{kDa}$ corresponding to the glycosylated form. Molecular weights are calculated using protein standards. $(B)$ Densitometry of the $57 \mathrm{kDa}$ form of EMMPRIN from the western blots scanned for the 3 groups of patients ( idiopathic pulmonary fibrosis $\mathrm{n}=12$; $\boldsymbol{\square}$ diffuse and parenchymal lung disorders, $\mathrm{n}=8$; $\square$ individual patients with evidence of fibrosis on computed tomography; $\boldsymbol{\Delta}$ without diffuse and parenchymal lung disorders, $\mathrm{n}=6$ ). Results are expressed as a percentage of the intensity of a band of a common reference sample loaded onto each gel (relative intensity). IPF: idiopathic pulmonary fibrosis; DPLD: diffuse parenchymal lung disorders; ns: not statistically significant.

\section{DISCUSSION}

This study reported the expression of EMMPRIN in IPF and in interstitial and fibrotic lung disorders not associated with IPF. We have observed that EMMPRIN was present to a greater extent in the bronchoalveolar lavage fluids of fibrosis patients compared to subjects without interstitial 


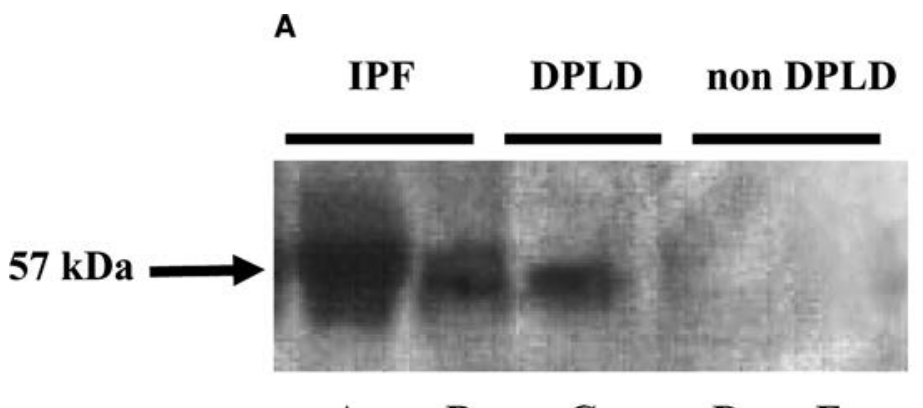

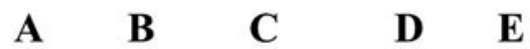

B

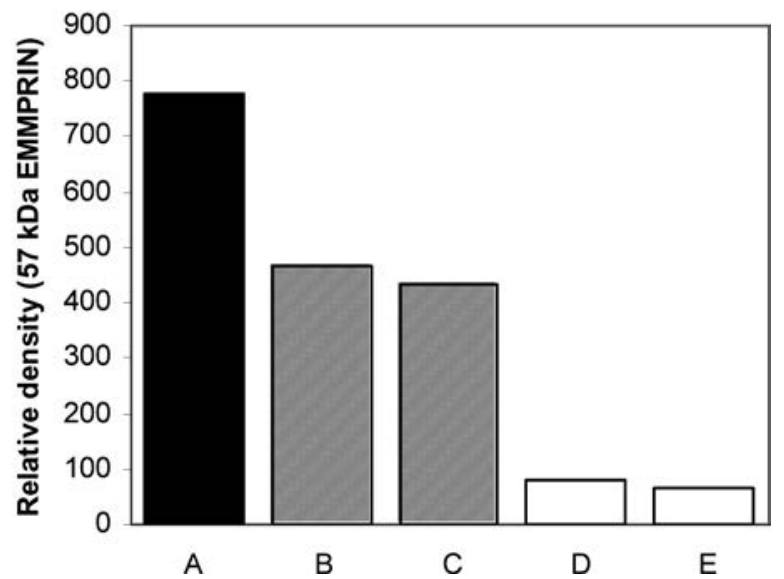

FIGURE $3(A)$ Western blot of EMMPRIN on cell lysates and culture supernatants of alveolar macrophages (AMs) recovered from BAL of 3 patients: 1 with IPF (lane A), 1 with a diffuse parenchymal lung disorder (DPLD) (lanes B and C), and 1 without DPLD (lanes D and E). AMs were harvested at 24 hours. Only the full-glycosylated form of EMMPRIN at $57 \mathrm{kDa}$ was observed. Lanes A, C, E: culture supernatants of AMs; lanes B and D: cell lysates. $(B)$ Results of the intensity of the band on Western blot expressed in relative density.

or fibrotic lesions. Then, we aimed to precise the cellular sources of EMMPRIN and relationships between EMMPRIN and fibrosis. Two cellular sources of EMMPRIN appear to be important in lung fibrosis. The alveolar macrophages from fibrotic patients spontaneously produce EMMPRIN. Furthermore, the specific expression of EMMPRIN in the pneumocytes close to fibrosis areas in IPF suggests the potential involvement of the alveolar epithelial cells in fibrogenic lung processes by secreting EMMPRIN.

The present findings suggest that EMMPRIN expression is directly linked to fibrosis because of the high expression of EMMPRIN in BAL of patients with IPF and with DPLD. Moreover, we observed on UIP sections an intense staining of EMMPRIN in the areas of fibrosis (honeycomb areas 
or fibroblastic foci), whereas areas of normal lung parenchyma did not express EMMPRIN. In this context, Betsuyaku and colleagues [26] also observed the presence of EMMPRIN in fibrotic area-associated macrophages and bronchiolar epithelium in an experimental model of bleomycin-induced pulmonary fibrosis in mice.

In agreement with our observations, several data support the presence of EMMPRIN in alveolar macrophages. Indeed, in normal lung, EMMPRIN has been detected in alveolar macrophages [15, 25]. Moreover, the expression of EMMPRIN in alveolar macrophages is increased in diverse lung pathologic processes including lung fibrosis in mice [26]. Here, we observed that EMMPRIN was spontaneously produced by alveolar macrophages from fibrotic patients (IPF and DPLD) and these results suggest that alveolar macrophages from fibrotic patients could produce more EMMPRIN both in the extracellular medium and inside the cells themselves than alveolar macrophages from nonfibrotic lung. Moreover, the cell surface localization of EMMPRIN has also been demonstrated by our studies. Thereby, it is hypothesized that EMMPRIN acts on cells either by direct cell contact or by its soluble fraction obtained from the total glycoprotein by proteolytic cleavage or by vesicular shedding [13, 32]. Regarding the cell-cell interactions, both macrophage-fibroblast and macrophagemacrophage interactions are possible cause of MMP and EMMPRIN production. In this context, the ability of EMMPRIN to stimulate its own production within the same population of cells was previously demonstrated in primary lung fibroblasts with a positive-feedback regulation of EMMPRIN gene expression [24, 33].

Tissue macrophages may also play an important role in producing EMMPRIN. Indeed, we observed by immunohistochemistry that activated tissue macrophages present in granulomas in sarcoidosis expressed EMMPRIN with an intense staining. Moreover, the 2 DPLD patients with sarcoidosis had high levels of EMMPRIN in their BAL fluid. It suggests that EMMPRIN is mainly produced by activated tissue macrophages. However, we did not found any correlation between EMMPRIN levels in the BAL fluids and the number of alveolar macrophages, probably because tissue macrophages may synthesize and secrete an important part of EMMPRIN, mostly in interstitial lung diseases such as sarcoidosis.

One other possible source of EMMPRIN could be bronchiolar epithelial cells. Indeed, it was previously reported that EMMPRIN is expressed in bronchiolar epithelial cells of current smokers and of bleomycin-treated mice [25, 26]. Accordingly, we also observed EMMPRIN expression in bronchiolar epithelial cells of all fibrosis and nonfibrosis patients.

The present study clearly demonstrates the presence of EMMPRIN in type II pneumocytes close to inflammation and fibrosis on UIP sections, suggesting an important role for these cell types in IPF disorder. Following 
alveolar damage, activated alveolar epithelial cells can release many factors such as profibrogenic cytokines, transforming growth factor- $\beta_{1}$, tumor necrosis factor- $\alpha$, and platelet-derived growth factor, leading to fibroblast migration and proliferation and changes in cell phenotype $[4,5]$. Fibroblasts may provoke basement membrane disruption and alveolar cell apoptosis. The presence of EMMPRIN in pneumocytes associated with inflammation and fibrosis suggests that activated alveolar epithelial cells synthesize and secrete EMMPRIN, which could act on neighboring fibroblasts through the basement membrane defects by inducing their metalloproteinase production.

However, the presence of EMMPRIN in non-DPLD patients is not a surprise, because some of them present diseases such as carcinomas or left ventricular myocardium failure, which could explain the presence of EMMPRIN in their BAL fluid [15-20, 22]. Moreover, tobacco use is a condition that could influence the presence of EMMPRIN [25], even though we did not observe any correlation between the presence of EMMPRIN and tobacco use in our patients. Several studies reported a minimal EMMPRIN expression in healthy lung, mainly in alveolar macrophages and in bronchiolar epithelial cells $[12,16]$.

In conclusion, in lung fibrosis, both the inflammatory (macrophages) and the epithelial (bronchiolar and alveolar) cells may release and activate a number of mediators, including EMMPRIN, that activates fibroblasts secreting several MMPs. This should lead consequently to the accumulation and remodeling of the extracellular matrix leading to pulmonary fibrosis. Determining the interactions between cellular sources of EMMPRIN and their potential target cells is required to further investigate the potential role of EMMPRIN in lung fibrosis.

\section{REFERENCES}

[1] Allen-Cooper J: Pulmonary fibrosis. Pathways are slowly coming into light. Am J Respir Cell Mol Biol. 2000;22:520-523.

[2] American Thoracic Society: Idiopathic pulmonary fibrosis: diagnosis and treatment. International consensus statement. Am J Respir Crit Care Med. 2000;161:646-664.

[3] American thoracic society/European respiratory society. International multidisciplinary consensus. Classification of the idiopathic interstitial pneumonias. Am J Respir Crit Care Med. 2002;165:277-304.

[4] Selman M, King T, Pardo A: Idiopathic pulmonary fibrosis: prevailing and evolving about its pathogenesis and implications for therapy. Ann Intern Med. 2001;134:136-151.

[5] Selman M, Pardo A: Idiopathic pulmonary fibrosis: an epithelial/fibroblastic cross-talk disorder. Respir Res. 2002;3:1-8.

[6] Hayashi T, Stelter-Stevenson W, Fleming M, Fishback N, Koss M, Liotta L, Ferrans V, Travis W: Immunohistochemical study of metalloproteinases and their tissue inhibitors in the lungs of patients with diffuse alveolar damage and idiopathic pulmonary fibrosis. Am J Pathol. 1996;149:1241-1256.

[7] Lemjabbar H, Gosset P, Lechapt-Zalcman E, Franco-Montoya ML, Wallaert B, Harf A, Lafuma C: Overexpression of alveolar macrophage gelatinase B (MMP-9) in patients with idiopathic 
pulmonary fibrosis. Effects of steroid and immunosuppressive treatment. Am J Respir Cell Mol Biol. 1999;20:903-913.

[8] Henry MT, McMahon K, Mackarel AJ, Prikk K, Sorsa T, Maisi P, Sepper R, Fizgerald MX, O’Connor CM: Matrix metalloproteinases and tissue inhibitor of metalloproteinase-1 in sarcoidosis and IPF. Eur Respir J. 2002;20:1220-1227.

[9] Lagente V, Boichot E, Corbel M, Artola M, Caulet-Maugendre S, Planquois J-P: Role of gelatinase (MMP-2 and MMP-9) activity in acute lung injury and pulmonary fibrosis. Curr Top Pharmacol. 2003;7:93-99.

[10] Fukuda N, Ishizaki M, Kudoh S, Kitaichi M, Yamanaka: Localization of matrix metalloproteinases$1,-2$, and -9 and tissue inhibitor of metalloproteinase-2 in interstitial lung diseases. Lab Invest. 1998;78:687-698.

[11] Ellis S, Nabeshima K, Biswas C: Monoclonal antibody preparation and purification of a tumor cell collagenase-stimulatory factor. Cancer Res. 1989;49:3385-3391.

[12] Muramatsu T, Miyauchi T: Basigin (CD147): a multifunctional transmembrane protein involved in reproduction, neural function, inflammation and tumor invasion. Histol Histopathol. 2003;18:981-987.

[13] Gabison EE, Hoang-Xuan T, Mauviel A, Menashi S: EMMPRIN/CD147, an MMP modulator in cancer, development and tissue repair. Biochimie. 2005;87:361-368.

[14] Biswas C, Zhang Y, DeCastro R, Guo H, Nakamura T, Kataoka H: The human tumor cell-derived collagenase stimulatory factor (renamed EMMPRIN) is a member of the immunoglobulin superfamily. Cancer Res. 1995;55:434-439.

[15] Polette M, Gilles C, Marchand V, Lorenzato M, Toole B, Tournier JM, Zucker S, Birembaut P: Tumor collagenase stimulatory factor (TCSF) expression and localization in human lung and breast cancers. J Histochem Cytochem. 1997;45:703-709.

[16] Caudroy S, Polette M, Tournier JM, Burlet H, Toole B, Zucker S, Birembaut P: Expression of the extracellular matrix metalloproteinase inducer (EMMPRIN) and the matrix metalloproteinase-2 in bronchopulmonary and breast lesions. J Histochem Cytochem. 1999;47:1575-1580.

[17] Taylor PM, Woodfield RJ, Hodgkin MN, Pettitt TR, Martin A, Kerr DJ, Wakelam MJ: Breast cancer cell-derived EMMPRIN stimulates fibroblast MMP-2 release through a phospholipase $\mathrm{A}_{2}$ and 5-lipoxygenase catalyzed pathway. Oncogene. 2002;21:5765-5772.

[18] Suzuki S, Sato M, Senoo H, Kazuo I: Direct cell-cell interaction enhances pro-MMP2 production and activation in co-culture of laryngeal cancer cells and fibroblasts: involvement of EMMPRIN and MT1-MMP. Exp Cell Res. 2002;28:55-68.

[19] Van den Oord J, Paemen L, Opdenakker G, De Wolf-Peeters C: Expression of gelatinase B and the extracellular matrix metalloproteinase inducer EMMPRIN in benign and malignant pigment cell lesions of the skin. Am J Pathol. 1997;151:665-670.

[20] Chen X, Kanekura T, Kanski T: Expression of basigin in human fetal, infantile and adult skin and in basal cell carcinoma. J Cutan Pathol. 2001;28:184-190.

[21] Major TC, Liang L, Lu X, Rosebury W, Bocan TM: Extracellular matrix metalloproteinase inducer (EMMPRIN) is induced upon monocyte differentiation and is expressed in human atheroma. Arterioscler Thromb Vasc Biol. 2002;22:1200-1207.

[22] Spinale F, Coker M, Heung L, Bond B, Gunasinghe H, Etoh T, Goldberg A, Zellner J, Crumbley J: A matrix metalloproteinase induction/activation system exists in the human left ventricular myocardium and is upregulated in heart failure. Circulation. 2000;102:1944-1954.

[23] Foda HD, Rollo E, Drews M, Conner C, Appelt K, Shalinsky DR: Ventilator-induced lung injury upregulates and activates gelatinases and EMMPRIN. Am J Respir Cell Mol Biol. 2001;25:717-724.

[24] Gabison EE, Mourah S, Steinfels E, Yan L, Hoang-Xuan T, Watsky MA, De Wever B, Calvo F, Mauviel A, Menashi S: Differential expression of extracellular matrix metalloproteinase inducer (CD 147) in normal and ulcerated corneas. Am J Pathol. 2005;166:209-219.

[25] Betsuyaku T, Tanino M, Nagai K, Nasuhara Y, Nishimura M, Semior R. EMMPRIN is increased in smokers' bronchoalveolar lavage fluid. Am J Respir Crit Care Med. 2003;24:26-60.

[26] Betsuyaku T, Kadomatsu K, Griffin GL, Muramatsu T, Senior R: Increased basigin in bleomycininduced lung injury. Am J Respir Cell Mol Biol. 2003;28:600-606.

[27] Li R, Huang L, Guo H, Tool B: Basigin (murine EMMPRIN) stimulates matrix metalloproteinase production by fibroblasts. FEBS Lett. 1998;441:88-92. 
[28] Sameshima T, Nabeshima K, Toole BP, Yokogami K, Okoda Y, Goya T, Koono M, Wakisaka S: Glioma cell extracellular matrix metalloproteinase inducer (EMMPRIN) stimulates production of membrane-type matrix metalloproteinases and activated gelatinase $\mathrm{A}$ in co-cultures with brain-derived fibroblasts. Cancer Lett. 2000;157:177-184.

[29] Sun J, Hemler ME: Regulation of MMP-1 and MMP-2 production through CD147/extracellular matrix metalloproteinase inducer interactions. Cancer Res. 2001;61:2276-2281.

[30] Kataoka H, DeCastro R, Zucker S, Biswas C: Tumor cell-derived collagenase-stimulatory factor increases expression of interstitial collagenase, stromelysin, and 72-kDa gelatinase. Cancer Res. 1993;53:3154-3158.

[31] Lim M, Martinez T, Jablons D, Cameron R, Guo H, Toole B, Li JD, Basbaum C: Tumor-derived EMMPRIN stimulates collagenase transcription through MAPK p38. FEBS Lett. 1998;441:88-92.

[32] Shidu S, Mengistab A, Tauscher A, La Vail J, Basbaum C: The microvesicle as a vehicle for EMMPRIN in tumor-stromal interactions. Oncogene. 2004;23:956-963.

[33] Tang Y, Kesavan P, Nakada MT, Yan L: Tumor-stroma interaction: positive feedback regulation of extracellular matrix metalloproteinase inducer (EMMPRIN) expression and matrix metalloproteinase-dependent generation of soluble EMMPRIN. Mol Cancer Res. 2004;2:73-80. 